\title{
Maternal Daily Intake of Polyphenol-Rich Dark Chocolate and Premature Closure of Ductus Arteriosus
}

\author{
Mayuka Anko ${ }^{1,2}$, Satoru Ikenoue ${ }^{1 *}$, Makoto Kawamura ${ }^{3}$ and Kazumi Yakubo ${ }^{1}$ \\ ${ }^{1}$ Department of Obstetrics and Gynecology, Saitama City Hospital, Japan \\ ${ }^{2}$ Department of Obstetrics and Gynecology, Keio University School of Medicine, Japan \\ ${ }^{3}$ Department of Obstetrics and Gynecology, Sannoh Clinic, Japan \\ *Corresponding author: Satoru Ikenoue, Department of Obstetrics and Gynecology, Saitama City Hospital, Japan
}

\begin{abstract}
ARTICLE INFO
Received: 幽 September 02, 2019

Published: 㹃 September 10, 2019

Citation: Mayuka A, Satoru I, Makoto K, Kazumi Y. Maternal Daily Intake of Polyphenol-Rich Dark Chocolate and Premature Closure of Ductus Arteriosus. Biomed J Sci \& Tech Res 21(2)-2019. BJSTR. MS.ID.003575.
\end{abstract}

Keywords: Fetal Ultrasound; PolyphenolRich Dark Chocolate; Premature Closure of Ductus Arteriosus; Third Trimester of Gestation

\section{ABSTRACT}

Premature closure of ductus arteriosus (PCDA) is known to be induced by antiinflammatory agents including polyphenols. Dark chocolates are widely consumed and rich in polyphenols. Here, we report a case of PCDA potentially associated with maternal daily intake of polyphenol-rich dark chocolate in late gestation. A 28-year-old primigravida patient was admitted in our hospital at 38 weeks because of non-reassuring fetal heart rate pattern. Fetal echocardiography revealed absent ductus arteriosus flow and tricuspid regurgitation. Because PCDA was suspected, caesarian section was performed to avoid consequent risk of fetal heart failure. Postnatal echocardiography performed immediately after birth confirmed tricuspid regurgitation, right ventricular hypertrophy, pulmonary hypertension and absent ductus arteriosus flow, confirming PCDA. The mother reportedly consumed three pieces of polyphenol-rich dark chocolate (127 mg polyphenol per piece) daily after 34 weeks. Pregnant women should be careful regarding their diet and monitor the consumption of polyphenol-rich foods, especially in the third trimester.

Abbreviations: A-Ao: Ascending Aorta; D-Ao: Descending Aorta; DA: Ductus Arteriosus; LV: Left Ventricle; RA: Right Atrium; RV: Right Ventricle; LVOT: Left Ventricular Outlet Flow; RVOT: Right Ventricular Outlet Flow

\section{Introduction}

Premature closure of ductus arteriosus (PCDA), defined as early constriction and closure of fetal ductus arteriosus, is induced by a certain class of anti-inflammatory agents (cyclooxygenase inhibitors), which inhibit prostaglandin synthesis. Prostaglandins, as a vasodilator, maintain patency of the fetal ductus arteriosus. Because the ductus arteriosus directs $80-85 \%$ of the right ventricular output, constriction of the ductus arteriosus could lead to persistent pulmonary hypertension, right ventricular heart failure, hydrops, and subsequent fetal demise [1]. Polyphenols are known to inhibit the enzymes cyclooxygenase and the subsequent transformation of arachidonic acid into prostaglandins [2]. Here, we report a case of PCDA potentially associated with maternal daily intake of polyphenol-rich dark chocolate in the third trimester of gestation.

\section{Case Report}

A 28-year-old primigravida woman was admitted in our institution at 38 weeks of gestation because of decreased fetal movement and a non-reassuring fetal heart rate pattern (absence of acceleration and occasional late deceleration). Before admission, her prenatal course had been unremarkable. Fetal echocardiography at 30 weeks of gestation (performed because of an unsatisfactory study in mid-gestation) confirmed normal cardiac structures and normal right-to-left flow through the patent ductus arteriosus. However, detailed fetal evaluation, including fetal ultrasonography on admission at 38 weeks, revealed absent ductus arteriosus flow (Figure 1A) and moderate tricuspid regurgitation. (Figure 1B) (Supplement Video) Based on these findings, premature closure 
of ductus arteriosus (PCDA) was suspected. The right ventricle was slightly dilated; however, the fetus showed no evidence of subcutaneous edema, pericardial effusion, ascites, or cardiomegaly. Doppler flow velocimetry of the umbilical cord and ductus venosus were within normal range. The biophysical profile score was 6/10 (non-reactive heart rate pattern, absence of fetal breathing movement). The mother was not in labor and the uterine cervix was closed. The decision to deliver via emergency caesarian section was taken to avoid consequent risk of fetal heart failure and hydrops. The neonate weighed $3312 \mathrm{~g}$ with Apgar scores of 8 and 9 at 1 and 5 minutes, respectively.

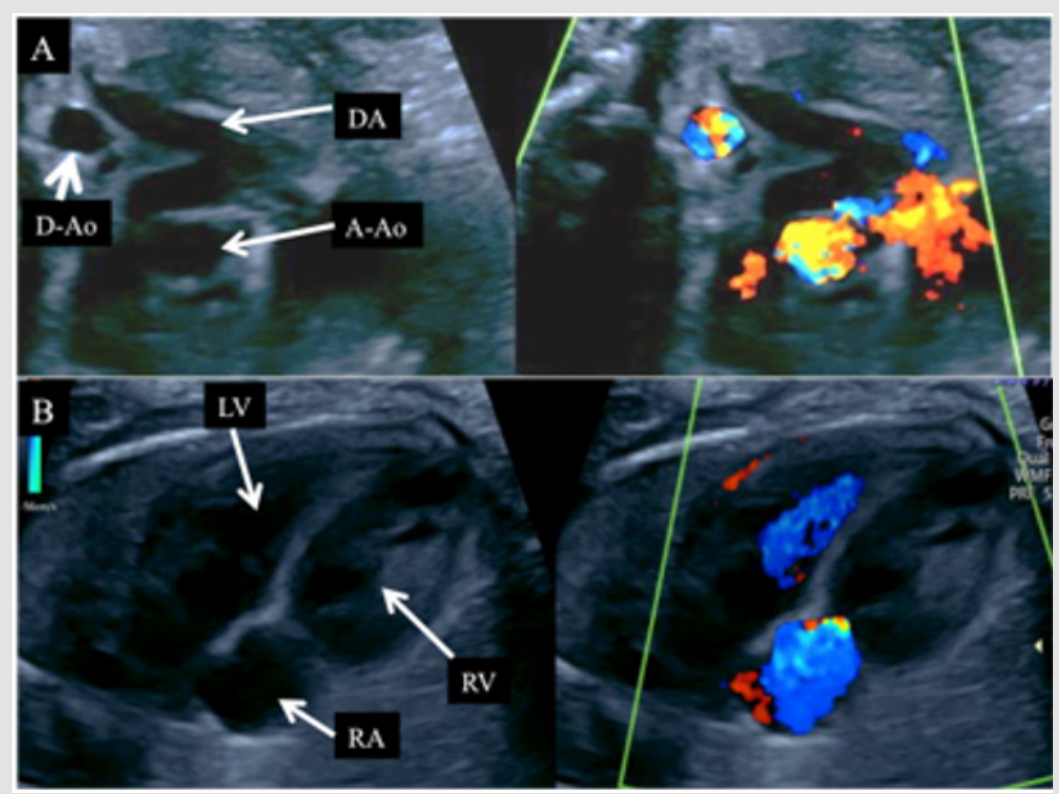

Figure 1: Fetal echocardiography and color doppler flow at 38 weeks of gestation.

A) Note the absence of ductus arteriosus flow

B) Tricuspid regurgitation.

Supplement Video

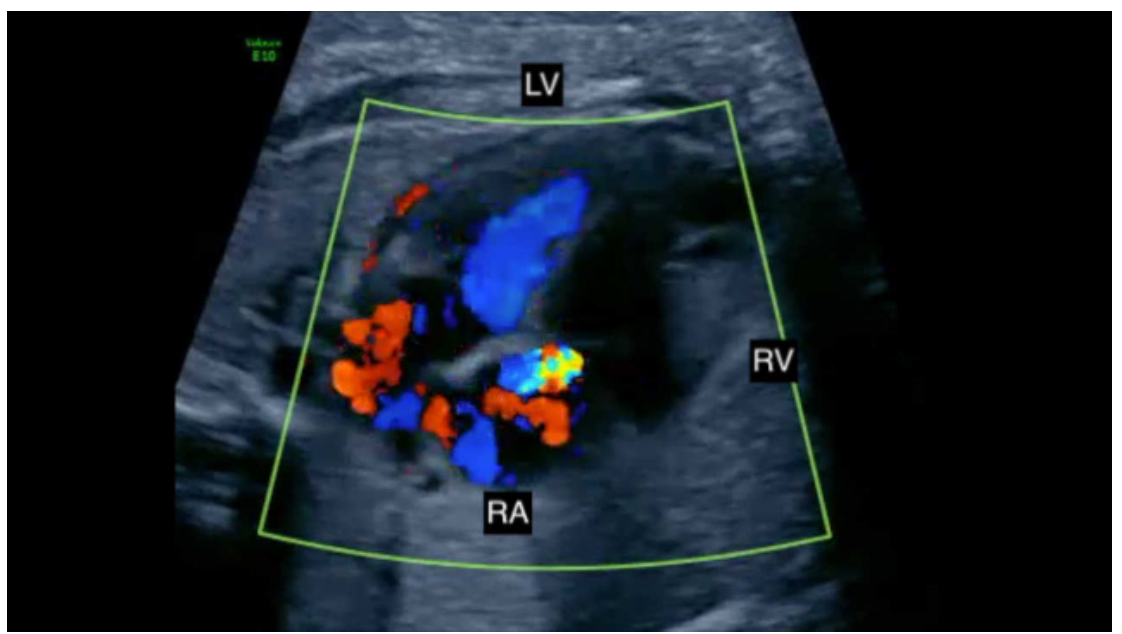

Postnatal echocardiography performed immediately after birth confirmed tricuspid regurgitation, right ventricular hypertrophy, pulmonary hypertension, and absent ductus arteriosus flow, confirming PCDA. The neonate was transferred to the neonatal intensive-care unit and placed on nasal high-flow oxygen and intravenous vasodilators for pulmonary hypertension. These were discontinued on postnatal day 3 , and the neonate was discharged on postnatal day 8 without complications. The mother denied taking any medications during pregnancy, including non-steroidal anti-inflammatory drugs. Detailed maternal dietary assessment revealed absence of polyphenol-rich foods, including berries, apples, sweet cherries, plums, green tea, or black tea [3]. However, she reportedly consumed three pieces of polyphenol-rich dark chocolate (127 mg polyphenol per piece) daily after the $34^{\text {th }}$ week of gestation.

\section{Discussion}

The present case of PCDA was possibly associated with maternal daily intake of polyphenol-rich dark chocolate after 34 weeks. Ductus arteriosus closure was confirmed on fetal echocardiography 
performed both before the constriction of ductus arteriosus (at 30 weeks) and after the closure of this vessel (at 38 weeks). The fetal ductus arteriosus at 38 weeks was interrupted completely, which possibly contributed to tricuspid regurgitation. Echocardiography performed immediately after birth also reconfirmed a constricted ductus arteriosus with right ventricular hypertrophy, tricuspid regurgitation, and pulmonary hypertension. Cesarean section performed soon after diagnosis and neonatal intensive care immediately after birth improved the postnatal course. Several reports have documented PCDA cases after maternal abundant polyphenol consumption. Daily intake of prune berry and violet vegetable juice (containing anthocyanins) for the last ten days before delivery was associated with PCDA at 38 weeks of gestation [4]. Maternal daily intake of fresh oranges (rich in flavanones) also induced constriction of ductus arteriosus at 30 weeks, which ameliorated by cessation of orange intake [5].

The ductus arteriosus becomes more sensitive to constrictive factors with increase in gestational age. The sensitivity to constricting factors (e.g. indomethacin) is estimated to be $5-10 \%$ at 27 weeks, $15-20 \%$ at 32 weeks, and almost $100 \%$ at 34 weeks [6], which is compatible with the present case. To date, there is no consensus concerning the amount of polyphenol consumption that could cause ductal constriction. The present case is characterized to describe the amount of maternal polyphenol intake from polyphenol-rich dark chocolate (381 mg/day) that was taken along with the daily diet after 34 weeks. Zielinsky et al. conducted a prospective study investigating the polyphenol dose that could induce PCDA [7]. A hundred and two fetuses exposed to polyphenolrich foods (daily estimated maternal consumption $>75^{\text {th }}$ percentile, or $1098 \mathrm{mg}$ ) were compared with 41 unexposed fetuses (daily estimated maternal consumption $<25^{\text {th }}$ percentile, or $127 \mathrm{mg}$ ). Mean peak systolic and diastolic velocities of ductus arteriosus and the mean ratio of right ventricle to left ventricle were significantly higher in the exposed fetuses. The present case lacks complete evaluation of the mother's total polyphenol intake. However, the regular Japanese diet has been reported to provide approximately $853 \mathrm{mg}$ polyphenols/day [8], and the mother consumed the usual Japanese diet before delivery. Detailed dietary assessment revealed that the dark chocolate (381 mg polyphenol per day) was her only polyphenol intake, and she possibly consumed $47 \%$ more polyphenols per day after 34 weeks of gestation. The present findings support previous reports regarding the dosedependent effect of polyphenols on the ductus arteriosus. Recently, polyphenols have become a popular functional food because of their antioxidant, anti-inflammatory, and anti-hypertensive effects, by inhibiting cyclooxygenase activity [2].
Polyphenols cannot be synthesized by humans and comprise several subgroups. Cacao contains numerous flavonoids [9]. Regular consumption of cacao chocolate is reported to reduce inflammatory markers and prevent hypertension and cardiovascular disease [2]. Based on these positive aspects, polyphenol-rich chocolate is now widely craved by women of reproductive age. However, the adverse effect of maternal intake of polyphenol-rich chocolate in late gestation, as in the present case, is not fully appreciated. These factors may increase the risk of maternal exposure to polyphenolrich chocolate in the third trimester of pregnancy and subsequent PCDA. Pregnant women should therefore be careful regarding their diet and monitor the consumption of flavonoid and polyphenol-rich foods, especially in the third trimester.

\section{Acknowledgement}

We thank all medical staffs in Saitama City Hospital who contributed to the excellent patient care for this study.

\section{Conflict of Interest}

The authors report no conflict of interest.

\section{References}

1. Gewillig M, Brown SC, De Catte L (2009) Premature foetal closure of the arterial duct: clinical presentations and outcome. Eur Heart J 30(12): 1530-1536.

2. Scalbert A, Johnson IT, Saltmarsh M (2005) Polyphenols: antioxidants and beyond. Am J Clin Nutr 81(Suppl 1): 215s-217s.

3. Perez-Jimenez J, Neveu V, Vos F, Scalbert A (2010) Identification of the 100 richest dietary sources of polyphenols: an application of the PhenolExplorer database. Eur J Clin Nutr 64(Suppl 3): S112-120.

4. Tanaka M, Miyakoshi K, Yamada M, Kadohira I, Minegishi K, et al. (2011) Functional foods for the fetus? Acta Obstet Gynecol Scand 90(10):11721173.

5. Rakha S (2017) Excessive Maternal Orange Intake - A Reversible Etiology of Fetal Premature Ductus Arteriosus Constriction: A Case Report. Fetal Diagn Ther 42(2): 158-160.

6. Zielinsky P, Busato S (2013) Prenatal effects of maternal consumption of polyphenol-rich foods in late pregnancy upon fetal ductus arteriosus. Birth Defects Res C Embryo Today 99(4): 256-274.

7. Zielinsky P, Piccoli AL Jr, Manica JL (2010) Maternal consumption of polyphenol-rich foods in late pregnancy and fetal ductus arteriosus flow dynamics. J Perinatol 30(1): 17-21.

8. Fukushima Y, Ohie T, Yonekawa Y (2009) Coffee and green tea as a large source of antioxidant polyphenols in the Japanese population. J Agric Food Chem 57(4): 1253-1259.

9. Ding EL, Hutfless SM, Ding X, Girotra S (2006) Chocolate and prevention of cardiovascular disease: a systematic review. Nutr Metab (Lond) 3: 2. 


\section{ISSN: 2574-1241}

DOI: 10.26717/BJSTR.2019.21.003575

Satoru Ikenoue. Biomed J Sci \& Tech Res

(c) (P) This work is licensed under Creative

Submission Link: https://biomedres.us/submit-manuscript.php

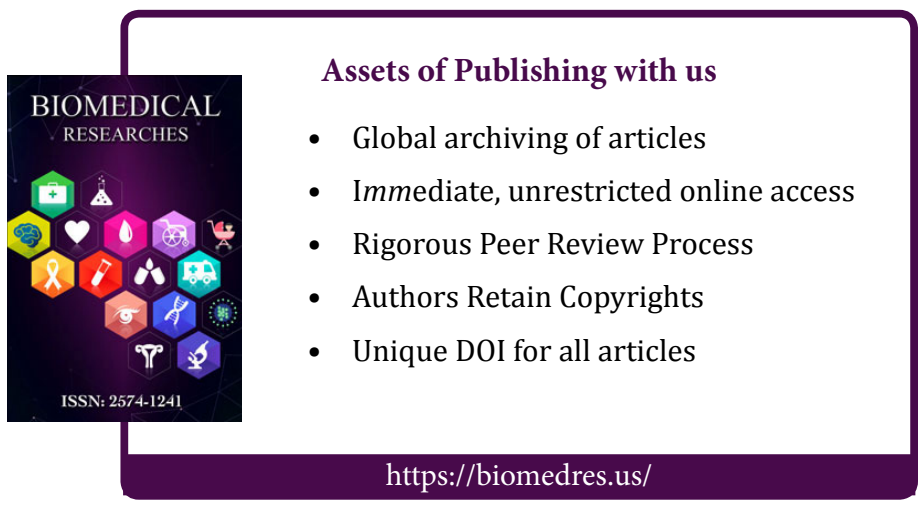

